\title{
Comparison of sniffing position and simple head extension for visualization of glottis during direct laryngoscopy.
}

\author{
Bhattarai B, Shrestha SK, Kandel S
}

Department of Anesthesiology,

Kathmandu University School of Medical Sciences,

Dhulikhel Hospital, Dhulikhel, Nepal

\section{Corresponding Author}

Dr. Basant Bhattarai

Department of Anesthesiology

Kathmandu University School of Medical Sciences

Dhulikhel Hospital, Dhulikhel, Nepal

E-Mail-basanbhattarai@yahoo.com

Citation

Bhattarai B, Shrestha SK, Kandel S. Comparison of sniffing position and simple head extension for visualization of glottis during direct laryngoscopy. Kathmandu Univ Med J 2011;33(1)58-63.

\begin{abstract}
Background

The ability of good glottis visualization during direct laryngoscopy is major determinant of easy tracheal intubation. Sniffing position for laryngoscopy is considered as gold standard and ideal position. Several studies have questioned the validation of sniffing potion.
\end{abstract}

\section{Objectives}

This study aims to compare relative efficacy of sniffing position and simple head extension for visualization of glottis during direct laryngoscopy.

\section{Methods}

Four hundred patients undergoing elective surgery under general anesthesia requiring endotracheal intubation were randomized into two groups and study was concluded. Glottic visualization was assessed using modified Cormack and Lehane classification. After laryngoscopy, tracheal intubation was performed and intubation difficulty scale was noted.

\section{Results}

Both the groups were comparable regarding glottis visualization. Both the groups were comparable in demographic profiles. All the intubation difficulty scale variables were comparable in both the groups except N3. Total Intubation Difficulty Score was better in sniffing position than in simple head extension group.

\section{Conclusion}

Glottic visualization and intubation difficulty score was better in sniffing position as compared to simple head extension.

\section{KEY WORDS}

sniffing position, simple head extension, direct laryngoscopy

\section{INTRODUCTION}

The ability to maintain glottis visualization during direct laryngoscopy is the major determinant of easy tracheal intubation. Correct positioning of the patient appears to be the main determining factor for obtaining good glottis visualization. Since Bannister and MacBeth introduced the "three-axe rule' 'in 1944, this position, as they proposed, might be obtained by slight flexion of the neck on the chest, whereas the optimal head position is extension at the plane of the face from the horizontal. ${ }^{1}$ This head position resembles a person "sniffing the morning air."Thus, the very common anesthesiologist's expression, "sniffing position," was born. The concept has been widely accepted as the foundation for understanding and teaching of direct laryngoscopy. The sniffing position for tracheal intubation is usually obtained by elevating the head with a blanket or pillow before induction. This maneuver is currently universally recommended, taught, and used throughout the anesthesia community. ${ }^{2-7}$ The sniffing position has been used for all these years. The first study of optimal patient positioning for orotracheal intubation was published in 1913 by Jackson stressing the importance of anterior flexion of the lower cervical spine, in addition to the more obvious extension of the atlanto- occipital joint. ${ }^{8}$

In 1999 Adnet et al after evaluating a radiograph obtained during intubation in sniffing position, concluded that although the sniffing position may provide the best laryngeal view, this is not due to the alignment of the three axes. This is an error perpetuated since 1944 that deserves 
reexamination. ${ }^{9}$ Similarly Chow $\mathrm{HC}$ pointed out that in majority of patients the tongue could be displaced more satisfactorily with satisfactory laryngeal exposure in a slight head extension position. He also observed that sniffing position does not permit total alignment of the three axes in conscious patients with normal airway patency. ${ }^{10}$

In the year 2001 Adnet et al further investigated the theory with the help of MRI scan to see if there was alignment of all the three axes. They found out that it was impossible to achieve a common axis and that there was no difference in the angle between simple head extension and sniffing position. They also concluded that sniffing position does not achieve alignment of the three important axes in conscious patients with normal airway anatomy. ${ }^{11}$ In the same year Adnet, in a different study, concluded that routine use of the sniffing position appears to provide no significant advantage over simple head extension for tracheal intubation in elective surgical patients. ${ }^{12}$ The sniffing position appears to be advantageous in patients who are obese or those with limited head extension.

In the view of above considerations the present study was designed to compare sniffing position with simple head extension for glottis visualization during direct laryngoscopy and ease of tracheal intubation in patients undergoing general anesthesia and endotracheal intubation.

\section{METHODS}

After approval by the institutional review committee of Dhulikhel Hospital - Kathmandu University Hospital and written informed consent from patients, 400 adult patients scheduled for elective surgeries under general anesthesia and endotracheal intubation were included in the study.

Randomization was performed by placing index cards labeled Group I- sniffing position and Group II- simple head extension into 400 sealed envelopes placed in random order, each group representing one method of intubation. At the time of a patient's enrollment, the next available envelope was placed with the patient's chart. Just before induction, the envelope was opened and the sequence thus determined.

All patients requiring general anesthesia with endotracheal intubation, aged between 20 to 60 years and ASA grades 1 and 2 were included. All patients with body mass index more than $30 \mathrm{~kg} / \mathrm{m}^{2}$, bucked teeth, restricted neck movement, inter- incisior gap less than $35 \mathrm{~mm}$, thyro- mental distance less than $65 \mathrm{~mm}$, with risk of regurgitation and aspiration, pharyngeal pathology and limitation of anterior and posterior movement of mandible were excluded.

Preoperative airway assessment was performed by an attending anesthesiologist. Inter- incisor gap was measured in millimeters with the mouth fully open. Thyro- mental distance was measured along a straight line from the thyroid notch to the lower border of the mandibular mentum with the head in full extension. Modified Mallampati classification was performed without phonation. ${ }^{13-14}$ Body mass index (BMI) was calculated as the weight in kilograms and divided by height in meters squared. Amplitude of neck and head movements was measured as described by Wilson et al: the patient's head and neck are fully extended, a pencil is placed on the forehead in alignment with the vertical axis and the patient is asked to fully flex while the anesthesiologist gauges the change in angle in reference to a fixed point. The angle is then classified according to two levels: less than $80^{\circ}$ and $80^{\circ}$ or more. ${ }^{15}$ Pathologic conditions associated with difficulties in laryngoscopy, such as malformation of the face, cervical spondylosis, tumors of the airway, longstanding diabetes, sleep apnea syndrome, limitation of mandibular anterior-posterior movement, and loose teeth, were also recorded.

Preoperative investigations were done based on surgical procedure, physical status and age of the patients. Patients were kept nil per oral for eight hours prior to the surgery and were given Diazepam, ten $\mathrm{mg}$ at night and five $\mathrm{mg}$ in the morning of the surgery.

After arrival in the operation theatre pre induction monitors, including noninvasive blood pressure monitoring, electrocardiography, and pulse oximetry were connected after securing the intravenous line. Before the induction of anesthesia all the Group I (Sniffing position) patients were placed supine and a cushioned wooden block of $8 \mathrm{~cm}$ height was placed under the head. At the time of laryngoscopy, the head was extended on the atlanto- occipital joint maximally. Group II (simple head extension) patients were placed supine without the wooden block. The head was extended maximally on the atlanto- occipital joint at the time of laryngoscopy.

Following pre oxygenation for three minutes, the standard induction technique was applied to all the patients which included Midazolam $0.03 \mathrm{mg} / \mathrm{kg}$, Fentanyl $2 \mu \mathrm{g} / \mathrm{kg}$, and Propofol titrated to loss of response to verbal commands. Neuromuscular blocker included $0.1 \mathrm{mg} / \mathrm{kg}$ of Vecuronium after ventilation with oxygen for three minutes; an independent anesthesiologist did a laryngoscopy in all the patients using three sized Macintosh laryngoscope blade to ensure the consistency of the technique. Glottic visualization during laryngoscopy was assessed by the same observer using modified Cormark and Lehane classification (without optimal external laryngeal manipulation). ${ }^{16}$ External laryngeal manipulation was permitted after evaluation in order to facilitate endotracheal intubation.

The "intubation difficulty scale"17 based on the seven parameters recorded by an independent observer was used to asses difficulty in intubation.

Intubation difficulty Scale(IDS) ${ }^{17}$

N1

0 -no supplementary attempt required

1 -any supplementary attempt required 
N2

0 -no supplementary operator required

1 - Any supplementary operator required

N3

0 - no alternative intubation technique used

1 -any alternative intubation technique used

N4

0 - Cormack \&Lehane Grade I

1- Cormack \&Lehane Grade II

2- Cormack \&Lehane Grade III

3- Cormack \&Lehane Grade IV

N5 Lifting Force During Laryngoscopy

0 - no subjectively increased lifting force required during laryngoscopy

1- Subjectively increased lifting force required during laryngoscopy

N6 External Laryngeal pressure for improved glottis visualization

0-no optimal external laryngeal manipulation required

1- Optimal external laryngeal manipulation required

N7 Position of Vocal cords at intubation

0 - vocal cords are abducted

1 - Vocal cords are adducted blocking the tube passage

2 Vocal cords not visualized

IDS is the sum of N1 to N7. Score $0=$ no difficulty at all., Score 1-5 = mild difficulty, Score $>5=$ moderate to severe difficulty.

After noting the grade of laryngoscopy, tracheal intubation was performed and intubation difficulty score was recorded.

Rest of the anesthesia was continued as per standard protocol. Post induction monitors included end tidal carbon dioxide and temperature. Anesthesia was maintained with Isoflurane and air in oxygen. At the end of procedure residual neuromuscular blocker was reversed with Neostigmine and Glycopyrolate. All the patients were extubated and shifted to post anesthesia care unit. Complications, like fall of peripheral oxygen saturation and dysrhythmias during laryngoscopy, were also noted.

Statistical analysis was done suing SPSS version 16. Unpaired t test was used for the age, sex and body mass index, mouth opening, hyo- mental distance, thyro- mental distance and sterno- mental distance. Chi square test was applied for assessing statistical significance of modified Mallampati grade, glottis visualization grade and intubation difficulty score. A P value of $<0.05$ was taken as significant.

Results.

Demographic Profile

Group I and II were comparable in demographic profile with respect to age, sex, height, weight and body mass index.
Table 1. Demographic profile in both the group of patients. Both the groups were comparable in demographic profiles. $(p>0.05)$

$\begin{array}{lcc}\text { Characteristics } & \text { Group I } & \text { Group II } \\ \text { Total Patients } & 200 & 200 \\ \text { Age } & 33.5 \pm 15 & 34.7 \pm 13 \\ \text { Sex M/F } & 135: 65 & 145: 55 \\ \text { Height } & 1.60 \pm 0.05 & 1.61 \pm 0.03 \\ \text { Weight } & 60.44 \pm 9.86 & 64.89 \pm 11.40 \\ \text { Body Mass Index } & 22.28 \pm 4.42 & 23.05 \pm 3.51 \\ \text { Unpaired t testP>0.05 } & & \end{array}$

A total of four hundred patients were evaluated out of which 280 (70\%) were male and 120 (30\%) were female.

Table 2. Modified Mallampatti Classification

\begin{tabular}{lcc|}
\hline Mallampatti Classification & Group I & Group II \\
\hline Grade I & $130(65 \%)$ & $135(67.5 \%)$ \\
\hline Grade II & $45(22.5 \%)$ & $35(17.5 \%)$ \\
\hline Grade III & $20(10 \%)$ & $26(13 \%)$ \\
Grade IV & $5(2.5 \%)$ & $4(2 \%)$ \\
\hline Total (N) & 200 & 200 \\
\hline
\end{tabular}

I

Table 3. Interincisior Gap/Hyomental Distance /Thyromental Distance and Sternomental Distance. Both the groups were comparable in all these factors. $(p>0.05)$

\begin{tabular}{lcc} 
Distance $(\mathrm{mm})$ & Group I & Group II \\
\hline Inter Incisor Gap & $48.4 \pm 0.74$ & $47.9 \pm 0.73$ \\
Hyomental Distance & $53.54 \pm 3.75$ & $53.56 \pm 3.73$ \\
Sternomental Distance & $168.90 \pm 14.82$ & $168.80 \pm 15.16$ \\
Thyromental Distance & $83.50 \pm 8.05$ & $83.68 \pm 6.09$
\end{tabular}

Unpaired ttest, $p>0.05$

Table 3 shows Interincisior Gap/Hyomental Distance /Thyromental Distance and Sternomental Distance. Both the groups were comparable in all these factors. $(p>0.05)$

Table 4. Laryngoscopic difficulty based on Cormack and Lehane classification.

$\begin{array}{lll}\text { Cormack and Lehane Grade } & \text { Group I } & \text { Group II } \\ \text { Grade } 1 & 133 & 119 \\ \text { Grade 2 } & 62 & 64 \\ \text { Grade 3 } & 4 & 17 \\ \text { Grade } 4 & 1 & 0 \\ \text { Total } & 200 & 200\end{array}$

Laryngoscopy was possible in all the patients. Glottic visualization in both the groups of patients was statistically comparable in two groups. $(p>0.05)$ 


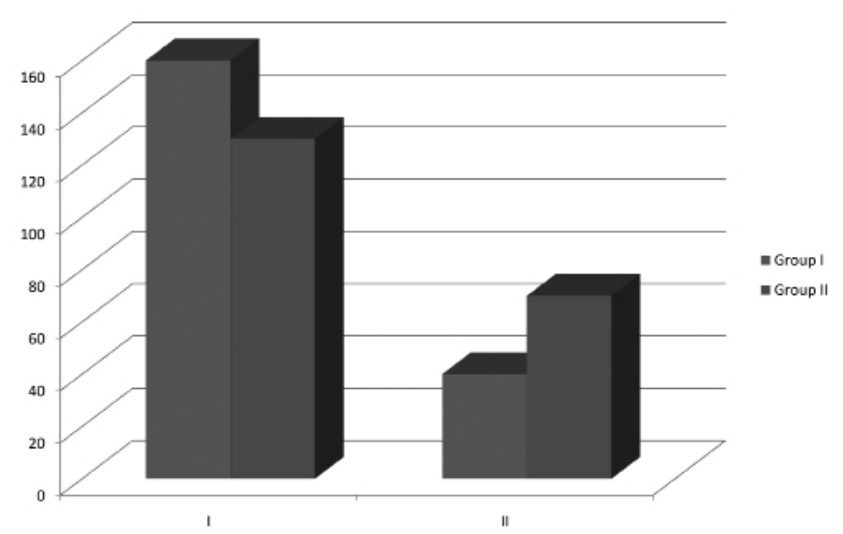

Figure 1. ASA physical status of the patients

Figure 1.160 patients in group I and 130 patients in group II belonged to ASA Grade I whereas 40 patients in group I and 70 patients in group 2 belonged to ASA Grade II physical status.

\section{INTUBATION DIFFICULTY SCALE}

Intubation difficulty scale variable N1 to N7 was statistically comparable in two groups. The percentage of intubation with and IDS score greater than 5 was $2 \%$ in simple head extension and $1 \%$ in sniffing position group.

Comparison of intubation difficulty scale between two groups demonstrated no statistically significant differences except in N3 which implies alternative technique required for intubation. However more patients in group II had N3 score of one $(n=44)$ as compared to group I $(n=20)(p<0.05)$. Similarly there were fewer patients in Group II $(n=156)$ than group I ( $n=180)$ with N3 score of zero $(p<0.05)$. The total IDS determining the ease of tracheal intubation was better in group I than group II $(p<0.05)$.

IDS of 0 corresponding easy intubation was observed in 116 patients in sniffing position as compared to 82 patients in simple head extension position $(p<0.05)$. IDS of $1-5$ corresponding to mild difficulty was seen in 82 patients in sniffing position and 114 in simple head extension $(p<0.05)$. Intubation difficulty score corresponding to moderate to severe difficulty was noted in ${ }^{2}$ patients in sniffing position and 4 patients in simple head extension ( $p>0.05$ ).

This implies that although the glottis visualization improved with the use of sniffing position, none of the two positions were advantageous over other for endotracheal intubation.

\section{DISCUSSION}

The sniffing position is universally recommended for oro- tracheal intubation in the operating room. Gillespie provided the first analysis of anatomical factors involves in laryngoscopy. According to him, the solution to the ease of intubation was to attain adequate depth of anesthesia and muscle relaxation conventional laryngoscopy and intubation requires a direct view of structures of larynx .

Jackson was first to emphasize the importance of position of head for laryngoscopy and intubation. ${ }^{8}$ The classical rationale for sniffing position is that the alignment of the mandibular axis, pharyngeal axis, and laryngeal axis is facilitated, permitting successful direct laryngoscopy. This alignment may be hypothetically obtained by flexing the neck on the chest and by elevating the head approximately 7-10 cm with a pad under the occiput (shoulders ordinarily remaining on the table). According to the theory, to bring the mandibular axis in line with both the pharyngeal and laryngeal axis, the head must also be extended on the neck (extension of the junction of the spine and skull (atlanto-occipital joint). ${ }^{4}$ This maneuver appears to be the fundamental first step before direct laryngoscopy. The article by Bannister and MacBeth ${ }^{1}$ is the only published experimental study to our knowledge that has attempted to provide an anatomic explanation and justification for use of this position. Nonetheless, the concept of three-axis alignment has been almost universally accepted.

Simple head extension position is attained by extending the head on the neck at the atlanto- occipital joint without placing a head ring under the head.

On evaluation of the radiographs taken during endotracheal intubation, Adnet et al concluded that there was no alignment of all three axes. He also investigated in healthy individuals with MRI and concluded that it was impossible to achieve the alignment of the entire three axes in sniffing position. ${ }^{9-11}$ Chow HC further investigated the concepts of three axes and concluded that there is only involvement of two axes "oral and pharyngeal" and "the tongue". ${ }^{10}$ All these studies however pointed out that the angle between laryngeal axis and the line of vision was decreased in sniffing as well as simple head extension position. Thus these positions are comparable among themselves but better than neutral position. ${ }^{11}$

This study was done to validate the benefit of the systemic use of sniffing position as compared to simple head extension for patients undergoing elective surgeries under general anesthesia with endotracheal intubation. The blade size was standardized for consistency. Modified Mallampatti grade as suggested by S. Rao Mallampatti and modified by Samson has been the cornerstone in preoperative assessment of the airway. ${ }^{13-14}$ In our study both the groups were comparable regarding Mallampatti grade distribution.

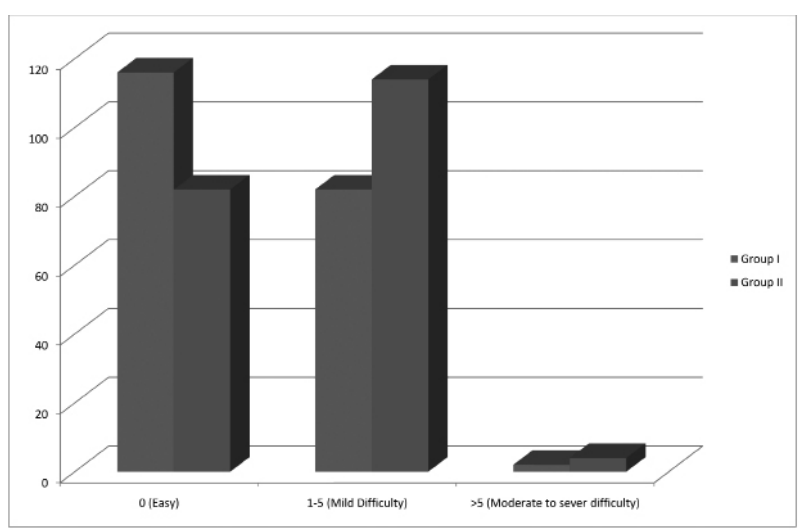

Figure 2. Comparision of Intubation difficulty scale in between two groups. 
Table 5. Intubation difficulty scale variables in both the group of patients.

\begin{tabular}{|c|c|c|c|c|c|c|c|c|c|c|c|c|c|c|c|c|}
\hline \multirow[b]{2}{*}{ Group I (N=200) } & \multicolumn{2}{|c|}{ N1 } & \multicolumn{2}{|c|}{ N2 } & \multicolumn{2}{|c|}{ N3 } & \multicolumn{4}{|c|}{ N4 } & \multicolumn{2}{|c|}{ N5 } & \multicolumn{2}{|c|}{ N6 } & \multicolumn{2}{|c|}{ N7 } \\
\hline & 194 & 6 & 196 & 4 & 180 & 20 & 116 & 70 & 12 & 2 & 172 & 28 & 160 & 40 & 198 & 2 \\
\hline Group II (N=200) & 186 & 14 & 196 & 4 & 156 & 44 & 102 & 84 & 14 & 0 & 174 & 26 & 140 & 60 & 198 & 2 \\
\hline$P$ value & \multicolumn{2}{|c|}{$>0.05$} & \multicolumn{2}{|c|}{$>0.05$} & \multicolumn{2}{|c|}{$<0.05^{*}$} & \multicolumn{4}{|c|}{$>0.05$} & \multicolumn{2}{|c|}{$>0.05$} & \multicolumn{2}{|c|}{$>0.05$} & \multicolumn{2}{|c|}{$>0.05$} \\
\hline
\end{tabular}

Mouth opening of less than two finger breadth or $35 \mathrm{~mm}$ is associated with difficult laryngoscopy and intubation. In the present study, mouth opening in both the groups was comparable. Our finding correlates well with the study by Singhalet al18. Difficult laryngoscopy should be considered if hyo- mental distance is less than $45 \mathrm{~mm}$ and thyromental distance is less than $60 \mathrm{~mm}$. In the present study all the patients had distance more than minimal values.

Although both groups were comparable regarding glottis visualization, the sniffing position was clinically better as compared to the simple head extension. Our results were similar to that of Adnet et al and Singhal et al. ${ }^{12-18}$

The present study has tried to assess a quantitative score-"The intubation difficulty scale" that can be used to evaluate the complexities of intubation. ${ }^{17}$ It is an objective scoring system, which is a function of seven parameters. An increased number of attempts N1 is the parameter most frequently described as being associated with difficult intubation. Introduction of second operator N2 or abandoning one technique for another N3 suggests a difficulty, perhaps more so than a simple additional attempt. As such changing operator or techniques implies two additional points: one for the change and one for the additional attempt.

The quality of laryngoscopic attempt is quantified using Cormack and Lehane classification. ${ }^{16}$ Intubation difficulty score is partly influenced by glottis exposure N4. However poor visualization of glottis is not always associated with a difficult intubation, thus the laryngoscopic quality alone is not an adequate measure of difficulty, but forms an important component of the intubation difficulty score.

Increasing lifting force and external laryngeal pressure are frequently used to improve the glottis exposure (N5 and N6). This score considers these two factors. Which tend to further emphasize the importance of quality of glottis visualization. Finally the status of the glottis exposure N7 will be affected by laryngospasm and cough, both of which have been identified as increasing the difficulty of intubation.

The total score of Intubation difficulty scale was better in patients with sniffing position as compared to head extension. As to the variable, both the groups were comparable except in N3 which included alternative intubation techniques like change in the blade or use of stylet. This shows that glottis is visualized in simple head extension but posterior part of larynx and esophagus come into view, also extension of head without elevation of occiput rotates the larynx anteriorly and increases the distance from lips to the glottis. So the use of alternative techniques is increased in patients with simple head extension.

This study's limitations included a limited series of patients, the fact that only Macintosh blade was used and if other blades were used, the results could be different, and the surrogate variables were not cross tested.

\section{CONCLUSION}

The results of the present study suggest that there is no significant difference between sniffing position and simple head extension with regards to incidence of better glottis visualization during direct laryngoscopy. However, the universal practice of sniffing position in anesthesiology teaching cannot be abandoned. Large multi- centric studies involving large group of patients may draw better conclusion than single center study with small group of patients.

\section{REFERENCES}

1. Bannister FB, Macbeth RG: Direct laryngoscopy and tracheal intubation. Lancet 1944; 2:651-4.

2. Stoelting RK: Endotracheal intubation, Anesthesia. Edited by Miller RD. New York, Churchill Livingstone, 1986, pp 523-32.

3. Finucane BT, Santora AH: Principles of Airway Management. St. Louis, Mosby, 1996.

4. Boisson-Bertrand D, Bourgain JL, Camboulives J, Crinquette V, Cros AM, Dubreuil M, Eurin B, Haberer JP, Pottecher T, Thorin D, Ravussin $P$, Riou B. Difficult intubation. French Society of Anesthesia and Intensive Care. A collective expertise. Ann FrAnesthRéanim 1996; 15(2):207-14.

5. Benumof JL: Conventional (laryngoscopic) orotracheal and nasotracheal intubation (single-lumen tube), Airway Management, Principles and Practices. Edited by Benumof JL. St Louis, Mosby, 1996, pp 261-76.

6. Murrin KR: Causes of difficult intubation and intubation procedure, Difficulties in Tracheal Intubation. Edited by Latto IP, Vaughan RS. London, Saunders, 1997, pp 89-106.

7. Schwartz DE, Wiener Kronish JP: Management of difficult airway. Clin Chest Med 1991; 12:483-95.

8. Jackson $\mathrm{C}$ : The technique of insertion of intratracheal insufflation tubes. SurgGynecolObstet 1913; 17:507-9.

9. Adnet $F$. The three axes alignment theory and the sniffing position perpetuation of an anatomic myth? Anesthesiology 1999; 91: 1964-5.

10. Chou HC. Rethinking the three axes alignment theory for direct laryngoscopy. ActaAnaesthesiolScand 2001;45:261-4. 
11. Adnet $F$. Study of the sniffing position by magnetic resonance imaging. Anesthesiology 2001; 94: 83-6.

12. Adnet F. Randomized study comparing the "sniffing position" with simple head extension for laryngoscopic view in elective surgery patients. Anesthesiology 2001; 95: 836-41.

13. Mallampati SR, Gatt SP, Gugino LD, Desai SP, Waraksa B, Freiberger D, Liu PL. A clinical sign to predict difficult tracheal intubation: a prospective study. Can AnaesthSoc J. 1985 Jul; 32(4):429-34.

14. Samsoon GL, Young JR. Difficult tracheal intubation: a retrospective study. Anaesthesia. 1987 May;42(5):487-90.

15. Wilson ME, Spiegelhalter $D$, Robertson JA, Lesser P. Predicting difficult intubation. Br J Anaesth. 1988 Aug; 61(2):211-6.

16. Cormack RS, Lehane J. Difficult tracheal intubation in obstetrics. Anesthesia 1984;39: 1105-1111.

17. Adnet F, Borron SW, Rachine SX, Clemessy JL, Fournier JL, Plaisence P, Lapandry C.The Intubation Difficulty Scale. Anesthesiology 1997; 87:1290-7.

18. Singhal SK, Malhotra N, Sharma S. Comparison of Sniffing Position and Simple Head Extension for Visualization of Glottis During DirectLaryngoscopy.Indian Journal of Anaesthesia 2008; 52 (5):546550. 